\title{
Control of Synchronization Regimes in Networks of Mobile Interacting Agents
}

\author{
Fernando Perez-Diaz, ${ }^{1, *}$ Ruediger Zillmer, ${ }^{2,3,}$ and Roderich Gro $\beta^{3, \$}$ \\ ${ }^{1}$ Department of Computer Science, The University of Sheffield, Sheffield S1 4DP, United Kingdom \\ ${ }^{2}$ Unilever R\&D, Port Sunlight CH63 3JW, United Kingdom \\ ${ }^{3}$ Department of Automatic Control and Systems Engineering, \\ The University of Sheffield, Sheffield S1 3JD, United Kingdom \\ (Received 9 May 2016; revised manuscript received 16 December 2016; published 5 May 2017)
}

\begin{abstract}
We investigate synchronization in a population of mobile pulse-coupled agents with a view towards implementations in swarm-robotics systems and mobile sensor networks. Previous theoretical approaches dealt with range and nearest-neighbor interactions. In the latter case, a synchronization-hindering regime for intermediate agent mobility is found. We investigate the robustness of this intermediate regime under practical scenarios. We show that synchronization in the intermediate regime can be predicted by means of a suitable metric of the phase response curve. Furthermore, we study more-realistic $K$-nearest-neighbor and cone-of-vision interactions, showing that it is possible to control the extent of the synchronizationhindering region by appropriately tuning the size of the neighborhood. To assess the effect of noise, we analyze the propagation of perturbations over the network and draw an analogy between the response in the hindering regime and stable chaos. Our findings reveal the conditions for the control of clock or activity synchronization of agents with intermediate mobility. In addition, the emergence of the intermediate regime is validated experimentally using a swarm of physical robots interacting with cone-of-vision interactions.
\end{abstract}

DOI: 10.1103/PhysRevApplied.7.054002

\section{INTRODUCTION}

Synchronization refers to a process by which two or more systems adjust one of their dynamical properties over time to achieve a coherent behavior by means of their interaction. As such, synchronization constitutes a pervasive concept in natural sciences, as well as in technological applications. Crickets chirping in unison, pacemaker cells firing rhythmically, the tide locking of astronomical bodies, and the coherence of laser beams are but a few examples of synchronization $[1,2]$.

Since the seminal work of Winfree [3] in the 1960s, a vast body of research has studied synchronization in complex networks (see Ref. [4] and the references therein for a comprehensive review). One approach to synchronization that has gathered much attention concerns models where the coupling between units is mediated by pulses. The work of Mirollo and Strogatz [5] pioneered the theoretical study of such pulse-coupled oscillators by determining various conditions under which a network of globally connected oscillators will always synchronize. Most of the subsequent work has focused on static

\footnotetext{
*fernando.perez.diaz@sheffield.ac.uk

†ruediger.zillmer.gb@ieee.org

r.gross@sheffield.ac.uk
}

Published by the American Physical Society under the terms of the Creative Commons Attribution 4.0 International license. Further distribution of this work must maintain attribution to the author(s) and the published article's title, journal citation, and DOI. networks [4]. However, in recent years, synchronization in temporal, or time-evolving, networks has received increasing attention, more specifically, in networks whose nodes represent physical agents that move in an environment and interact with local neighbors [6-17].

The study of such mobile pulse-coupled oscillators (MPCOs) aims to shed light on the effect that motioninduced topological changes have on synchronization. Oscillator mobility leads to novel behavior if the interaction is restricted to a local neighborhood, forming a time-evolving network. In general, agent mobility is necessary to achieve global synchronization. Most of the existent work deals with random geometric graphs, where two nodes are connected if the distance between each other is smaller than a certain range. It is found that, for integrate-and-fire oscillators with this kind of connectivity, synchronization occurs faster with higher agent mobility $[6,9,16]$. This monotonic dependence is what one would intuitively expect, given that, at high speeds, all agents interact with each other frequently, whereas at low speeds, the neighborhood of any particular agent does not often change, leading to a rapid local synchrony but requiring a longer time to achieve coherence at a global scale [15]. Prignano et al. [7] reported the counterintuitive result that, for nearest-neighbor connectivity, the dependence is no longer monotonic. While the behavior at slow and fast speeds remains the same, an intermediate regime appears where synchronization is inhibited.

The MPCO framework, of locally interacting moving agents, is of interest in a variety of contexts. For instance, in vertebrate somitogenesis, it was found that collective cell 
motion boosts synchronization of coupled genetic oscillators [18]. In technical domains, there has also been growing interest in MPCOs. As they operate at the physical layer by transmitting simple identical pulses, rather than packet messages, MPCOs are especially suitable in noisy or communication-limited environments $[11,16,19]$. Specifically, MPCOs have recently been studied in the contexts of mobile sensor networks [11,16,19], mobile ad hoc networks [11,20], and swarm-robotics systems $[15,21]$. Timing synchronization can be a prerequisite for the agents (robots, sensors, etc.) to achieve consensus and coordinate their activities. Certain modes of cooperative transport need synchronization to ensure that groups of robots push an object simultaneously [22,23]. Temporal synchronization has also been used in distributed sensing and data gathering by teams of robots [24,25]. For example, a swarm of robots is able to track a target that moves faster than any individual robot by synchronizing the timings of the robots' observations. Synchronization has also been proposed for power saving in sensor networks, by keeping the sensors' communication channels idle most of the time and using them only at precise times [26].

Conversely, desynchronization (i.e., temporal incoherence) has also been exploited in sensor networks in the context of task scheduling. By performing tasks at different times, monitoring activities or sleeping cycles can be organized and distributed [27,28]. Desynchronization can also benefit communication in this context, effectively implementing time-division medium access, a well-known medium-access-control protocol. By sending messages at different times, agents do not have to compete for the shared medium and can avoid message collisions [27-29].

In all of the preceding practical applications, one is confronted with several challenges. Examples are restricted communication range, latency, finite-amplitude perturbations due to noise or jitter, etc. However, only the range interaction has been studied in these practical contexts, and the effect of perturbations has not been considered.

This paper presents an in-depth analysis of the conditions and parameters that control the time required for a system of MPCOs to synchronize. We study the factors that affect the relationship between synchronization time and agent speed-from the monotonic dependence observed for range interactions to the nonmonotonic dependence in the nearest-neighbor case. Understanding the emergence of the aforementioned intermediate regime is of particular importance because it allows us to enable or impede synchronization depending on the application. In addition, we study the response of the system to perturbations such as the ones encountered in real systems. Furthermore, we validate experimentally the existence of the synchronization-inhibiting intermediate regime on a real system-a swarm of robots.

We start by analyzing the effect of the oscillator's phaseresponse curve on the nearest-neighbor scenario and find that the inhibitory regime can be eliminated by adding an appropriate refractory period-an interval of time after emitting a pulse in which pulses from other agents are not received or taken into account. This refractory period is indeed a necessity in real-world applications. Contrary to idealized systems, propagation of a signal through physical media incurs latency. The subsequent processing of this signal also involves delays. Moreover, wireless antennas and other communication devices may not simultaneously transmit and receive signals [16]. In a synchronized system, an oscillator can be pushed out of synchrony if it reacts to a slightly delayed signal from a neighbor. A refractory period can overcome problems that arise from these limitations.

Second, we analyze the effect of the neighborhood model, which determines the connectivity between agents. In practical applications, a range connectivity may not always be achievable. Furthermore, an agent may be incapable of determining whether it is the closest to another agent, rendering the nearest-neighbor interaction impractical. In this work, we study a more-realistic kind of connectivity, in which the oscillators are influenced by others only in their cone of vision. In a preliminary study [15], we reported that the dimensions of such a cone (namely, the angle and the radius), influence the synchronization time. For narrow or short cones of vision, the aforementioned nonmonotonic behavior is found. However, as the cone gets bigger, the intermediate regime disappears, and synchronization time monotonically decreases with agent speed. Equivalent results are presented for a system with $K$-nearest-neighbor connectivity, observing that, as $K$ increases, the intermediate regime becomes increasingly less inhibitory until the monotonic behavior is found.

Third, the effect of perturbations on a synchronized system is investigated. We show that the propagation of this perturbation in the intermediate regime displays characteristics akin to stable chaos [30]. In addition, we study a system with small clock differences (i.e., oscillators with slightly different oscillation periods) in order to model jitter or manufacturing variations.

Finally, we present experimental validation of some of our findings with a swarm of physical robots. Specifically, each robot possesses an internal oscillator and signals its firing by flashing onboard lights. The flashings can, in turn, be detected by other robots with their cameras. The field of view of the cameras effectively implements a cone of vision. The synchronization time of the robots displays a nonmonotonic dependence with respect to robot speed, which correlates with our simulation results.

This paper is organized as follows: In Sec. II, we introduce the utilized models and metrics. Section III describes the effect of the response curve. Section IV examines the effect of two neighborhood models on the synchronization curves. Section V investigates the effect of perturbations on the system. Section VI presents the experimental validation, and Sec. VII concludes the paper. 


\section{METHODS}

We consider a system of $N$ identical mobile agents moving in a two-dimensional (2D) bounded square environment of side length $L$.

The adjacency matrix of the network, $A(t)=\left\{a_{i j}(t)\right\}$, defines the neighborhood of each agent based on geometrical criteria. Owing to the mobility of the agents, the neighborhood evolves over time; $a_{i j}(t)=1$ if unit $j$ is in the neighborhood of unit $i$ at time $t$, and $a_{i j}(t)=0$ otherwise.

\section{A. Oscillator dynamics}

Each unit, $i$, is a simple integrate-and-fire oscillator with free-running dynamics,

$$
\frac{d \phi_{i}}{d t}=\frac{1}{\tau}
$$

where the phase $\phi_{i} \in[0,1]$ grows linearly in time with period $\tau$ (set here to 1 without loss of generality) until the threshold, 1, is reached and a firing event occurs. Upon firing, the oscillator resets its phase to 0 and sends an instantaneous pulse to its neighbors. The phase of neighboring oscillators is shifted by a certain amount, which depends on the timing of the incident signal.

Equation (2) represents the dynamics of the system at the moment of a firing of oscillator $i$ at time $t$,

$\phi_{i}\left(t^{-}\right)=1 \Rightarrow\left\{\begin{array}{l}\phi_{i}\left(t^{+}\right)=0 \\ \phi_{j}\left(t^{+}\right)=\phi_{j}\left(t^{-}\right)+a_{i j}(t) \Psi\left(\phi_{j}\left(t^{-}\right)\right)\end{array}\right.$

where the map $\Psi:[0,1] \rightarrow \mathbb{R}$ is called a phase-response curve (PRC). The factor $a_{i j}(t)$ guarantees that the phase shift is applied only to the current neighbors of the firing oscillator.

Several PRCs are considered: a multiplicative PRC, which produces an increase in phase proportional to the current phase $[6,7,21]$, and two PRCs, belonging to a class of response curves called delay-advance in the literature $[16,19,31]$, which cause a decrease (inhibition) of the phase for $\phi \leq 0.5$ and an increase (excitation) for $\phi>0.5$.

Equation (3) describes the multiplicative ( $\Psi_{\text {mult }}$ ) PRC, whereas Eqs. (4) and (5) describe the delay-advance PRCs, which we will call sawtooth $\left(\Psi_{\text {saw }}\right)$ and sine $\left(\Psi_{\text {sine }}\right)$, respectively:

$$
\begin{aligned}
& \Psi_{\text {mult }}(\phi)=\epsilon \phi, \quad \text { for } \phi \in(D, 1], \\
& \Psi_{\text {saw }}(\phi)=\left\{\begin{array}{ll}
-\kappa \phi, & \text { for } \phi \in(D, 0.5] \\
\kappa(1-\phi), & \text { for } \phi \in(0.5,1]
\end{array},\right. \\
& \Psi_{\text {sine }}(\phi)=-\kappa \sin (2 \pi \phi), \quad \text { for } \phi \in(D, 1] .
\end{aligned}
$$

Constants $\epsilon \in \mathbb{R}_{\geq 0}$ and $\kappa \in[0,1]$ characterize the strength of the interaction. The interval $[0, D]$, with
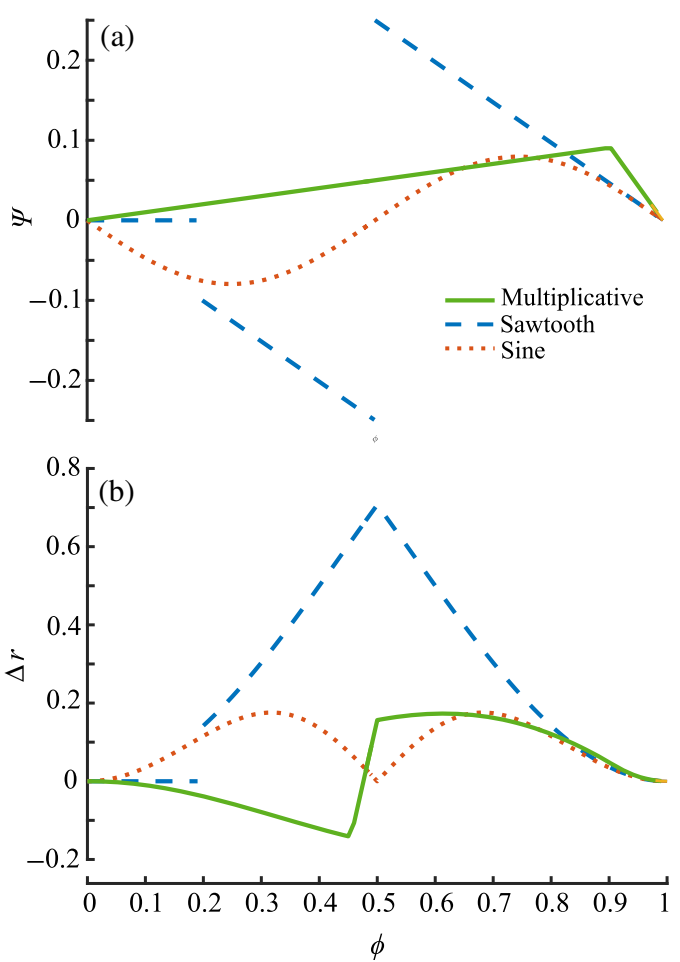

FIG. 1. (a) Multiplicative PRC with $\epsilon=0.1$ and $D=0$, sawtooth PRC with $\kappa=0.5$ and $D=0.2$, and sine PRC with $\kappa=0.08$ and $D=0$. (b) Corresponding order-parameter change, $\Delta r(\phi)$, after a firing of one oscillator in a two-unit fullyconnected system.

$0 \leq D<0.5$, is the refractory period, during which no phase update is produced $(\Psi=0)$. As mentioned in the Introduction, the refractory period is necessary in practical applications to counter physical delays, as well as other technical constraints. In all cases, the resulting phase after the update, $\phi\left(t^{+}\right)$, is capped at 1 in order to prevent overshooting. In other words, for any PRC, $\Psi$, the effective phase-response curve is $\Psi_{\text {eff }}(\phi)=\min (1-\phi, \Psi(\phi))$. Figure 1(a) shows examples of the three PRCs, where the aforesaid restriction has been taken into account.

Given that some units may fire upon receiving a phase update from a firing neighbor and, in turn, could elicit further firings, the phase shift is performed at frozen time until the phases of all oscillators have been updated. In addition, if more than one firing is simultaneously received by the same oscillator, a single phase update takes place.

\section{B. Motion dynamics}

The units are initially placed at randomly chosen positions within the bounded environment. Their initial orientations are chosen at random from $[0,2 \pi)$. Each unit moves in a straight line at constant speed $V$ until it reaches the environment boundary. At that point, the unit reorients to a direction randomly chosen from $[-(\pi / 2),(\pi / 2)]$ with respect to the boundary's normal and proceed with its 
straight-line motion in the new direction. All random numbers are generated using uniform distributions.

The chosen motion of the agents is decoupled from the dynamics of the associated oscillators. This is because synchronization is not the main objective of a robotic swarm or a sensor network but is instead a prerequisite for the agents to be able to coordinate their actions. Nevertheless, reorientation upon emitting or receiving a firing is also explored yielding qualitatively similar results (not presented here). In addition, whereas most previous work uses environments with cyclic boundary conditions $[6,7,17]$, we opt for a bounded environment for practical realism. Cyclic boundary conditions yield analogous results (not presented).

\section{Neighborhood models}

When an oscillator fires, it produces a phase shift on its neighbors. We consider two neighborhood models based on geometrical criteria: K-nearest-neighbor $\left(K_{\mathrm{nn}}\right)$ connectivity, and cone-of-vision (cone) connectivity.

Given the positions of the oscillators at time $t,\left\{\mathbf{X}_{\mathbf{i}}(t)\right\}$, the adjacency matrix of the network at that time, $A(t)$, is, in general, nonsymmetric (i.e., the graph is directed). The neighborhood of a given unit, $i$, at time $t$ is a set $\mathfrak{N}_{i}(t)$, which determines the nonzero elements of the adjacency matrix. Formally,

$$
a_{i j}(t)=\left\{\begin{array}{ll}
1 & \text { if } j \in \mathfrak{N}_{i}(t) \\
0 & \text { otherwise }
\end{array},\right.
$$

where $i, j \in\{1,2, \ldots, N\}$ and $i \neq j$. A unit cannot be its own neighbor; therefore, the adjacency matrix has all $0 \mathrm{~s}$ diagonal, $A_{i i}=0$.

In systems with $K$-nearest-neighbor connectivity, unit $i$ influences the $K$ other units that are spatially closest to itself, $K \in\{1,2, \ldots, N-1\}$. We have that the cardinality of the neighborhood, $\left|\mathfrak{N}_{i}^{K \mathrm{nn}}(t)\right|$ is $K$ and that

$$
j \in \mathfrak{N}_{i}^{K \mathrm{nn}}(t): \quad\left\|\mathbf{X}_{\mathbf{j}}-\mathbf{X}_{\mathbf{i}}\right\| \leq\left\|\mathbf{X}_{\mathbf{l}}-\mathbf{X}_{\mathbf{i}}\right\|, \quad \forall l \notin \mathfrak{N}_{i}^{K \mathrm{nn}}(t),
$$

where $\|\mathbf{X}\|$ represents the Euclidean norm. Note that the case where $K=N-1$ is the all-to-all connected network. Figure 2(a) shows an example of $K_{\mathrm{nn}}$ connectivity for $K=1$ in a system of three oscillators.

In systems with cone-of-vision connectivity, unit $j$ is considered the neighbor of another unit, $i$, if and only if $i$ lies inside the circular sector centered in $j$, with radius $R$ and angle $\theta$ and oriented in the direction of motion of $j$. That is,

$$
j \in \mathfrak{N}_{i}^{\text {cone }}(t), \quad \text { if }\left\{\begin{array}{l}
\left\|\mathbf{X}_{\mathbf{i}}-\mathbf{X}_{\mathbf{j}}\right\| \leq R \\
\frac{\mathbf{X}_{\mathbf{i}}-\mathbf{X}_{\mathbf{j}}}{\left\|\mathbf{X}_{\mathbf{i}}-\mathbf{X}_{\mathbf{j}}\right\|}\left\|\mathbf{V}_{\mathbf{j}}\right\| \\
\| \cos (\theta / 2)
\end{array},\right.
$$

where $\mathbf{V}_{\mathbf{j}}$ is the velocity vector of unit $j$, with $\left\|\mathbf{V}_{\mathbf{j}}\right\|=V$. Figure 2(b) shows an example of cone-of-vision connectivity for a system of three oscillators. (a)
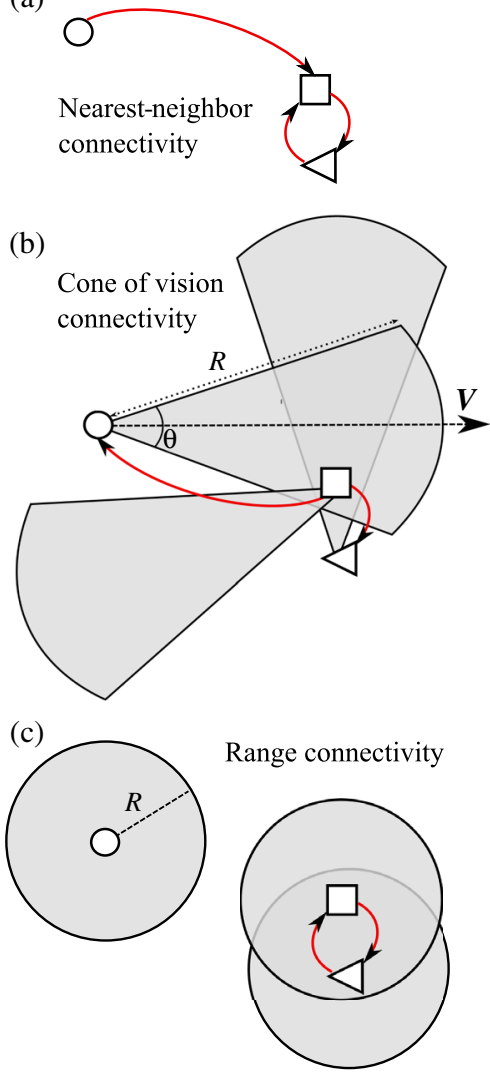

FIG. 2. Different neighborhood models and the corresponding interactions (the solid arrows) for a system of three units (open circle, open square, open triangle). The unit at the end of an arrow denotes the neighbor of the unit at its origin. (a) Nearest-neighbor connectivity. Each unit influences the spatially nearest unit. The square and the triangle influence each other, and the circle influences the square. (b) Cone-of-vision connectivity-if a unit can see another unit (see the text for details), the latter will influence the former. The square lies in the cone of vision of the other two units; thus, it influences both. The circle and the triangle do not influence other units because they do not lie in any other unit's cone of vision. (c) Range connectivity. Two units influence each other if they lie within the range $R$. The square and the triangle influence each other, while the circle does not influence other units, as they are out of range.

Note that, in the limit where $\theta \rightarrow 2 \pi$, the network becomes a random geometric graph, and the limit where $R \rightarrow \sqrt{2} L$ and $\theta \rightarrow 2 \pi$ corresponds to an all-to-all connected network.

It is important to remark on the directionality of the coneof-vision connectivity. Contrary to $K$-nearest-neighbor connectivity and the much-used range interaction, here, a firing oscillator, $i$, does not directly elicit a phase update on other oscillators. Instead, the recipient unit must be able to physically see $i$ to react to that firing [see Fig. 2(b)]. This choice is made to emulate a practical scenario. For instance, a robot may have only a directional sensor to detect the pulse [15]. The opposite directionality-that is, a cone of 
emission-is also investigated with similar results (not presented here). Note that the $360^{\circ}$ cone of emission is the previously studied range interaction and it is equivalent to the $360^{\circ}$ cone of vision [cf. Figs. 2(b) and 2(c)]. That is, if $i$ is in the $360^{\circ}$ cone of vision of $j, j$ will also be in the $360^{\circ}$ cone of emission of $i$.

\section{Synchronization metric}

The collective dynamics of the system is measured by the complex order parameter [4],

$$
r(t) e^{i 2 \pi \phi(t)}=\frac{1}{N} \sum_{j=1}^{N} e^{i 2 \pi \phi_{j}(t)},
$$

where $\phi(t)$ is the mean phase. Modulus $r(t)$ measures the level of coherence, from $r(t)=0$ for a totally incoherent system to $r(t)=1$ for complete synchronization. Henceforth, we will refer to the real part $r(t)$ as the order parameter without ambiguity.

A certain oscillator, $\phi_{1}$, is selected as a reference, and the order parameter is calculated upon its $k$ th firing at time $T_{k}$, $r\left(T_{k}\right)$. The simulation is stopped once the system is synchronized [i.e., $r\left(T_{k}\right)=1$ ] and the number of firings, $k$, emitted by the reference oscillator until synchronization is counted. This value, which we will call the synchronization time, $T_{\text {sync }}$, is a good measure of the time needed to achieve coherence independently of the oscillation period.

\section{E. Simulations}

Unless otherwise stated, all averages presented in the following sections are performed over 100 random initializations of positions, orientations, and phases for a population for $N=20$ units moving in an environment of size $L=100$. In order to study the dependence of the synchronization time on agent speed, 30 exponentially spaced speeds in the range $\left[10^{-2}, 10^{2}\right]$ are considered.

\section{EFFECT OF THE RESPONSE CURVE}

We start by extending Prignano et al.'s work [7] on oscillators interacting with their nearest neighbor to different PRCs. In their work, they observe a nonmonotonic dependence of the synchronization time $T_{\text {sync }}$ on the speed of agents $V$ for the multiplicative PRC, with no refractory period $(D=0)$. We observe that both delay-advance response curves, sawtooth and sine, display a monotonically decreasing dependence (see Fig. 3).

We attribute the observed difference of behavior to the sign of $\Psi(\phi)$. As can be seen in Fig. 1(a), both delayadvance response curves have a negative sign for $\phi<0.5$ and a positive sign otherwise. Consequently, the phase of an oscillator receiving a pulse will always get closer to the emitting pulse (note that the two phase limits, $\phi=0$ and $\phi=1$, are equivalent). However, for the multiplicative

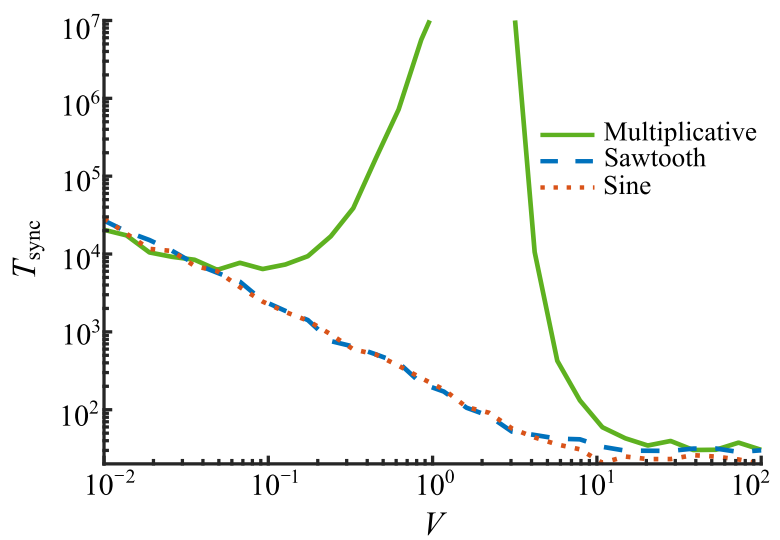

FIG. 3. Average time $T_{\text {sync }}$ (in number of cycles) required to synchronize 20 units with nearest-neighbor connectivity and moving with speed $V$ in a $2 \mathrm{D}$ square environment of side 100 for the multiplicative $(\epsilon=0.1)$, sawtooth $(\kappa=0.1)$, and sine $(\kappa=0.1)$ PRCs with no refractory period $(D=0)$.

PRC, the receiving oscillator will distance its phase from the pulse emitter for any $0<\phi<[1 /(2+\epsilon)]$.

We can measure the effect on synchronization of a PRC by considering a system of only two units, one emitting a pulse (i.e., phase equal to 1) and the other, with phase $\phi$, receiving it, and calculating the difference of the system's order parameter before and after the firing,

$$
\Delta r(\phi)=r\left(\phi\left(t^{+}\right)\right)-r\left(\phi\left(t^{-}\right)\right)
$$

Figure 1(b) shows that $\Delta r(\phi)$ is always positive for the delay-advance PRCs, but it is negative when $0<\phi<$ $[1 /(2+\epsilon)]$ for the multiplicative PRC. Adding a refractory period as in Eq. (3) confirms that this negative interval hinders synchronization (see Fig. 4). When suppressing the effects of this interval, the nonmonotonicity disappears as $D$ increases. Moreover, sufficiently large refractory periods

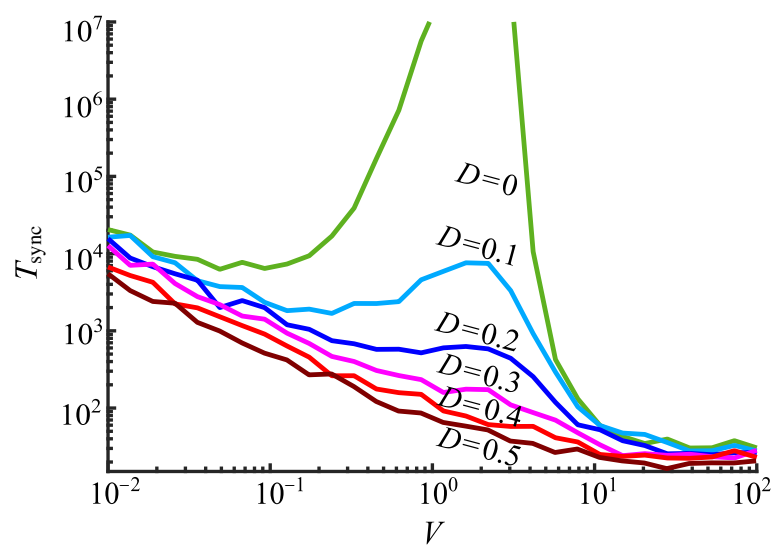

FIG. 4. Average time $T_{\text {sync }}$ (in number of cycles) required to synchronize 20 units with nearest-neighbor connectivity and moving with speed $V$ in a 2D square environment of side 100 using the multiplicative PRC for different refractory periods $D$. 


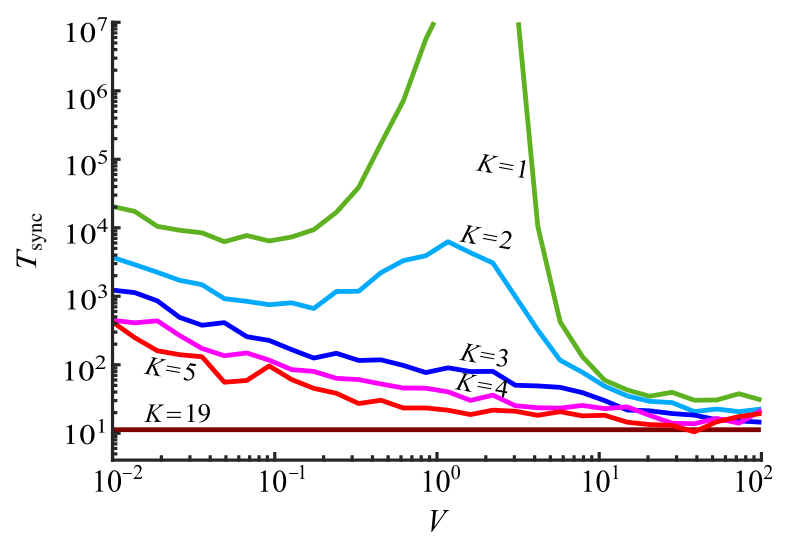

FIG. 5. Average time $T_{\text {sync }}$ (in number of cycles) required to synchronize 20 units with $K$-nearest-neighbor connectivity and moving with speed $V$ in a 2D square environment of side 100.

$(D \geq 0.4)$ display a faster synchronization for slow speeds than any of the delay-advance PRCs in Fig. 3.

\section{EFFECT OF THE NEIGHBORHOOD MODEL}

In the previous section, we concluded that the multiplicative PRC with no refractory period displays the strongest nonmonotonic behavior in a system with nearest-neighbor connectivity. In this section, we study the effect of two other neighborhood models for the same phase-response curve. Henceforth, we fix the multiplicative factor in Eq. (3) to $\epsilon=0.1$.

\section{A. $K$ nearest neighbors}

First, we extend the nearest-neighbor interaction to $K$ nearest neighbors. We observe that the intermediate, synchronization-inhibiting, regime becomes weaker as $K$ increases, until it finally disappears (see Fig. 5). A large enough $K(K>5)$ yields a monotonically decreasing dependence of $T_{\text {sync }}$ with respect to $V$, similar to the one observed in previous work for a $360^{\circ}$ range interaction [6].

It is worth noting that, in general, mobility is necessary for the system to synchronize. For a static population of oscillators, it is necessary (although typically not sufficient) for the undirected graph to form a single connected component for global synchronization to be achieved. Otherwise, two or more clusters could remain isolated from each other, reaching only local synchrony. The smallest cluster size with $K_{\mathrm{nn}}$ connectivity is the complete (fully connected) $(K+1)$-directed graph. We have that, for $K+1 \leq N / 2$, there could exist two or more noninteracting clusters and, therefore, synchronization is not guaranteed if the units are static. The case where $K=19$ in Fig. 5 corresponds to the trivial all-to-all connected network.

\section{B. Cone of vision}

In the preceding subsection, we observed how the synchronization time of the system can be tuned, not only by changing the speed of agents but also by changing the number of neighbors with which each unit interacts. This tuning is also applicable to a cone-of-vision neighborhood. The cone of vision, being a circular sector, can be considered an extension of the widely studied range interaction. However, in contrast to the latter, where $T_{\text {sync }}$ always exhibits a monotonic dependence on $V$, the dimensions of the cone of vision can alter the synchronization behavior.
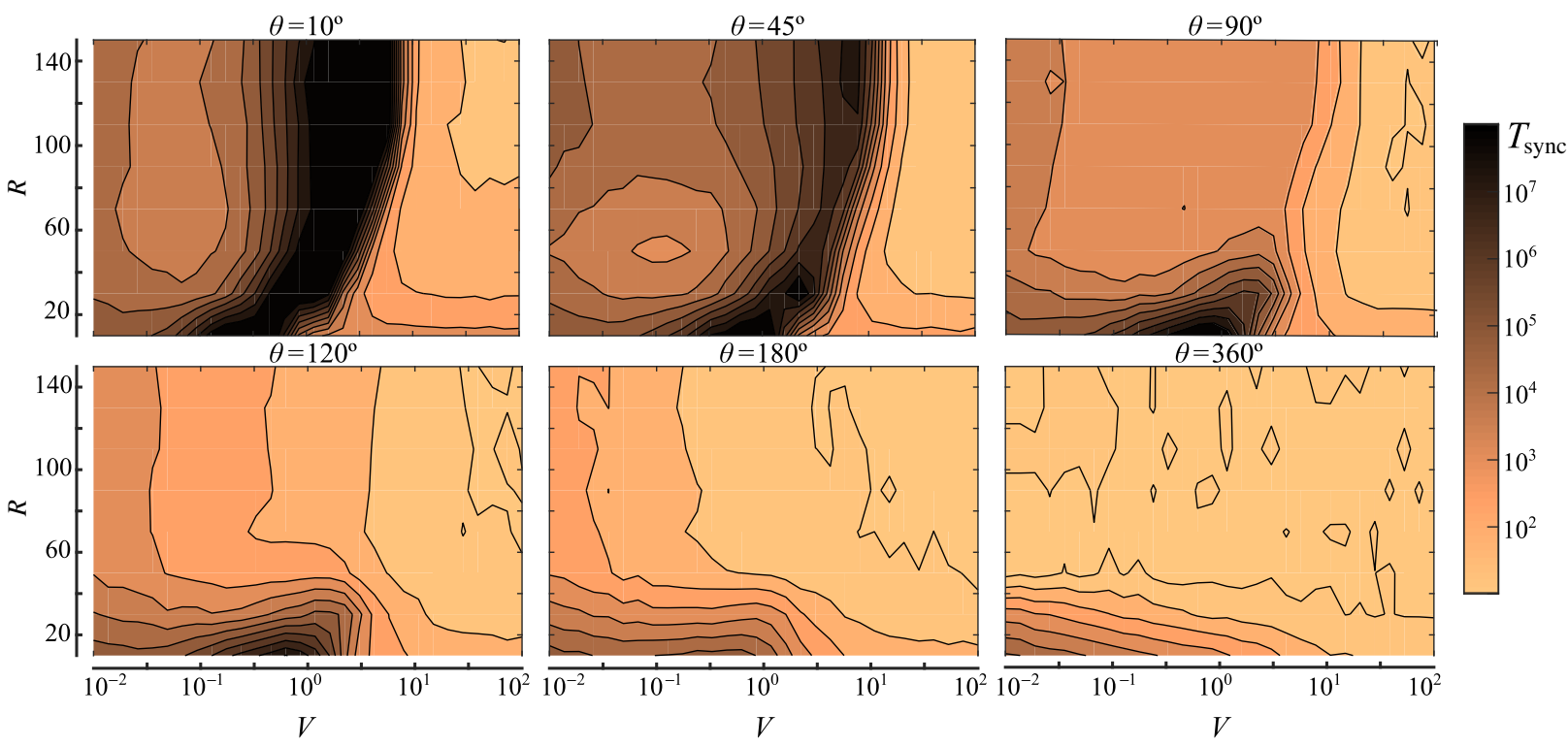

FIG. 6. Average time $T_{\text {sync }}$ (in number of cycles) required to synchronize 20 units with cone-of-vision connectivity (with radius $R$ and angle $\theta$ ) and moving with speed $V$ in a 2D square environment of side 100 using the multiplicative PRC. Eight equispaced radii are considered in the displayed interval. 
Figure 6 shows the resulting synchronization behavior for various cone angles and radii. Large $(R \rightarrow \sqrt{2} L)$ and wide $(\theta \rightarrow 2 \pi)$ cones lead to a decreasing dependence of $T_{\text {sync }}$ on $V$. Nevertheless, as the size of the cone decreases (either $\theta$ or $R$ decreases), the monotonicity is broken, and synchronization is slowed for an intermediate range of speeds. In fact, in the top-left panel in Fig. 6, three clearly distinct sectors, corresponding to three dynamical regimes, can be observed.

Note that the interplay between the radius and the angle of the cone is not trivial. For a given speed, a wider angle and larger radius produce, typically, faster synchronization. However, a greater radius can slow synchronization for particular speeds and angles (see Fig. 6 for $\theta=10^{\circ}, 45^{\circ}$, $90^{\circ}$, and $120^{\circ}$ ), or a wider angle can slow synchronization for particular speeds and radii (cf. Fig. 6 for $\theta=10^{\circ}$ and $120^{\circ}$ at $V=10^{\circ}$, with $R<20$ ).

As in the previous section, in the general case, mobility is necessary for the system to synchronize. For a cone of vision, only $R \geq \sqrt{2} L$ and $\theta=360^{\circ}$ (i.e., the all-to-all connected network) would guarantee that static units form a single connected cluster.

\section{EFFECT OF PERTURBATIONS}

Real-world systems are subject to a number of imperfections, such as noise and variations within manufacturing tolerances. In this section, we study the effect of perturbations in two ways: First, we measure the propagation of finite-amplitude perturbations through the system. Second, we study a system with slightly misaligned clocks to model possible physical faults or jitter.

\section{A. Finite-amplitude perturbations}

Departing from a fully synchronized state, one of the oscillators is initially displaced by a small but finite amount $\left[\phi_{1}(0)=0.001, \phi_{j \neq 1}(0)=0\right]$. Figure 7 provides a typical example of the effect of this perturbation over the system for a relatively small cone of vision $\left(R=40\right.$ and $\left.\theta=20^{\circ}\right)$. The figure shows the phase difference $|\Delta \phi|$ between the perturbed and unperturbed $\left[\phi_{1}(0)=0\right]$ systems over time.

In the slow regime (top panel), the perturbation spreads among the connected units, but the system settles before the topology changes. In the fast regime (bottom panel), the perturbation is quickly transferred to all other units. However its magnitude remains small until it completely vanishes due to the rapid changes in connectivity. The connection between two given oscillators is too short lived for long-lasting changes to be produced. In addition, because of the high speeds, the oscillators effectively experience a mean field of the interactions, which causes the displaced system to rapidly return to coherence. Naturally, the resulting synchronous state for both slow and fast regimes could be displaced by a constant amount with respect to the unperturbed scenario (see Fig. 7, bottom panel).

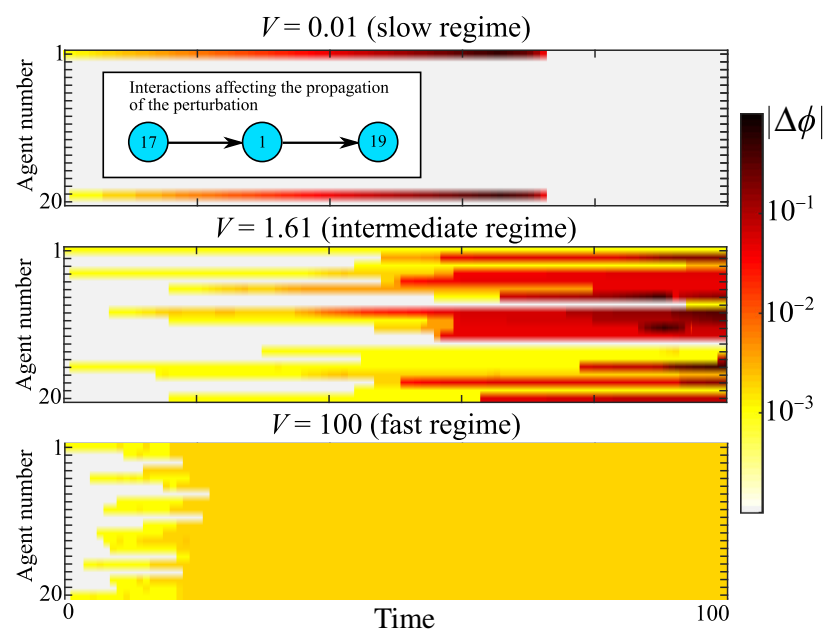

FIG. 7. Propagation of small perturbation over time in a system of 20 units with cone-of-vision connectivity $(R=40$ and $\theta=20^{\circ}$ ) using the multiplicative PRC. The color represents the magnitude, in logarithmic scale, of the phase difference, $|\Delta \phi|$, with respect to the unperturbed system. (Top panel) Slow regime. (Center panel) Intermediate regime. (Bottom panel) Fast regime. (Inset) Oscillators that play a role in the propagation of the perturbation in the slow regime. The initially perturbed unit 1 displaces unit 19. In parallel, undisturbed unit 17 brings 1 (and therefore 19) back to synchrony with the rest of the system.

In the intermediate regime (center panel), characteristics from both the slow and fast regimes are present. On the one hand, the movement is sufficiently fast for the perturbation to spread over all units. On the other hand, the changes of connectivity occur at a rate that still permits the magnitude of this perturbation to be amplified.

The obtained results are equivalent to those found for oscillators interacting with their nearest neighbor, as can be seen in Fig. 8. The plot shows that $|\Delta \phi|$ (the order parameter, $r$ ) increases (decreases) with time for intermediate speeds, whereas the perturbation vanishes after some time in the slow regime, and the system is barely affected in the fast regime.

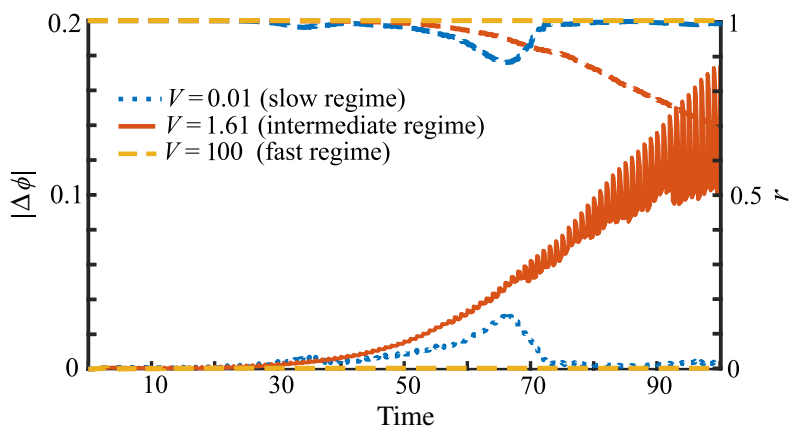

FIG. 8. Average evolution of the order parameter, $r$ (top curves), and $|\Delta \phi|$ (bottom curves) after applying a perturbation once to a single oscillator in an initially synchronized system of 20 units with nearest-neighbor connectivity. 
In the intermediate regime, all memory of the initially synchronized state is lost in the span of a few firing events, which is typical of chaotic and stochastic regimes. Synchrony will eventually be reached, however, after a long number of cycles, as if it were a randomly initialized system.

The sensitivity to small but finite-amplitude perturbations in the intermediate regime resembles the behavior of stable chaos, which has been observed in networks of integrate-and-fire neurons [30,32]. In contrast to standard chaos, stable chaos is a transient phenomenon restricted to finite time scales. However, the transient duration diverges exponentially with system size. Indeed, as shown in Fig. 9 for a system with nearest-neighbor interaction, the time to achieve synchronization after applying a perturbation grows exponentially with population size for intermediate speeds. In contrast, $T_{\text {sync }}$ barely depends on $N$ in the slow and fast regimes. Reference [7] found that the speed at which the system enters the fast regime for a nearestneighbor interaction is $V_{f} \propto L / \sqrt{N}$. Therefore, in Fig. 9, the density of the system, $N / L^{2}$, is kept constant, while the number of units is increased in order to ensure that the fastregime limit remains fixed.

\section{B. Clock jitter}

In order to model jitter, we consider a system where the oscillation period is different for each oscillator. Therefore, Eq. (1) transforms into

$$
\frac{d \phi_{i}}{d t}=\frac{1}{\tau_{i}}
$$

where $\tau_{i}$ is the oscillator period of unit $i$. In the following, $\tau_{i}$ is randomly selected from the Gaussian distribution centered in $\tau=1$ and with standard deviation $\sigma=5 \times 10^{-5}$. The choice of $\sigma$ is made to better illustrate the effect of clock misalignment on synchronization.

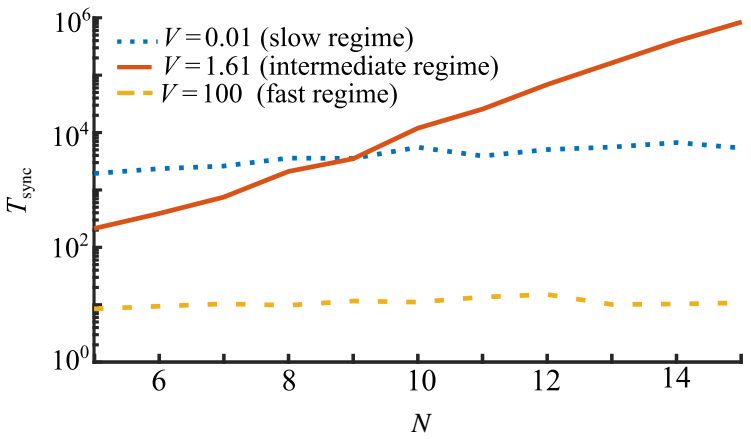

FIG. 9. The average transient time until synchronization, $T_{\text {sync }}$, as a function of the system size after a small perturbation is applied once to a single oscillator in an initially synchronized system with nearest-neighbor connectivity. An average is performed over 20 random initializations (positions and orientations) with a fixed initial perturbation $\left[\phi_{1}(0)=0.001, \phi_{j \neq 1}(0)=0\right]$.

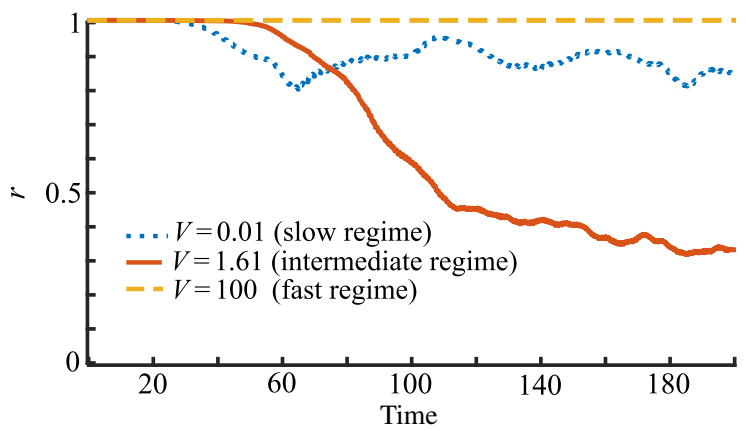

FIG. 10. Average evolution of the order parameter $r$, in a system with clock jitter, in which the oscillators are initially synchronized. Average over 100 populations of 20 units, with Gaussian distributed oscillator periods, $\tau_{i}$ (see the text for details).

Departing from a fully synchronized state, the evolution of the system is recorded. Figure 10 shows the order parameter over time for speeds in the three dynamical regimes in a system with nearest-neighbor connectivity. Similarly to the results reported in Sec. VA, the fast regime is negligibly affected by the perturbations. However, for both low and intermediate speeds, the oscillators are permanently displaced from synchrony. In the intermediate regime, the system gets totally desynchronized, almost approaching a random system $(r=0)$. By contrast, for slow speeds, connected oscillators can achieve local synchronization. Therefore, the effect on global synchronization is not as severe as in the intermediate regime.

\section{EXPERIMENTAL VALIDATION}

Implementation in a real-world system entails further challenges, such as the finite size of the agents, signal delays or failures, etc. Experiments with a swarm of physical robots are performed to validate our findings regarding the cone of vision neighborhood (Sec. IV B). (This section provides a summary of the experiments. Further details may be found in Ref. [33].)

\section{A. Setup}

The chosen robotic platform is the e-puck [34], a twowheeled cylindrical robot of $7.4 \mathrm{~cm}$ in diameter [Fig. 11(a)]. The e-puck is equipped with a ring of red light-emittingdiode (LED) lights, eight proximity infrared sensors, and a camera located at its front, offering a field of view (cone of vision) of $56^{\circ}$.

Ten e-pucks are placed in a square arena having sides measuring $L=10 \mathrm{~cm}$, with randomly initialized positions uniformly selected from a grid of equispaced positions with four possible starting orientations. The robots move in straight lines and reorient at the boundaries, as in the simulations. However, they also actively avoid collisions with other robots by using the proximity sensors.

Each robot has an internal oscillator with period $\tau=5 \mathrm{~s}$, also initialized at random. The robot signals the completion 
(a)

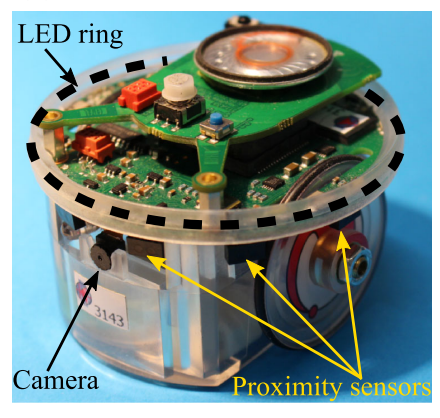

\section{(b)}

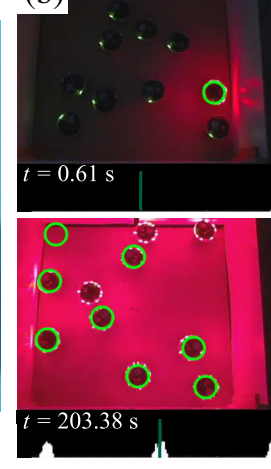

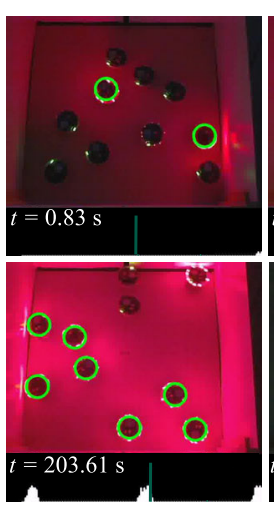

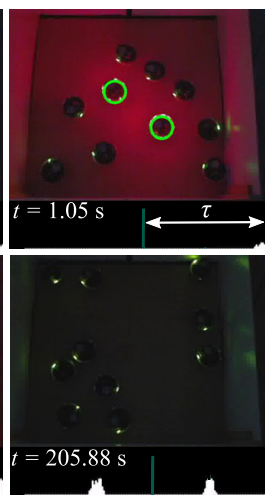

FIG. 11. (a) The e-puck robot. (b) Snapshots of the experimental setup with 10 e-puck robots in a square arena of side length $L=60 \mathrm{~cm}$. Robot flashings (active LED rings) are detected and superimposed as green circles (N.B., some flashings might occasionally be misclassified, as in the bottom-left frame). The lower strip shows an evolving histogram of the number of flashings detected over time. The system is considered to be synchronized if the distribution of the histogram around the current peak has a standard deviation smaller than two frames. of a cycle of its oscillator by activating its red LED ring for a short duration $(375 \mathrm{~ms})$. This flashing can, in turn, be detected by other robots with their cameras. A discrete version of Eq. (1) is implemented, using the multiplicative PRC of Eq. (3) with a refractory period $D=0.15(750 \mathrm{~ms})$.

\section{B. Results}

Figure 11(b) shows snapshots taken from a typical experiment with the e-puck robots. Tracking software detects the number of flashing robots at a given time (the green circles). Thus, a histogram of the number of firings per captured frame can be traced [see the lower strip in each frame of Fig. 11(b)]. Synchrony is measured as a function of the variance of the distribution within a period $\tau$. The smaller the variance, the greater the synchrony.

Figure 12 displays the synchronization time $T_{\text {sync }}$ of the system as a function of robot speed over a total of 50 trials. Despite the differences with the ideal simulated scenario,

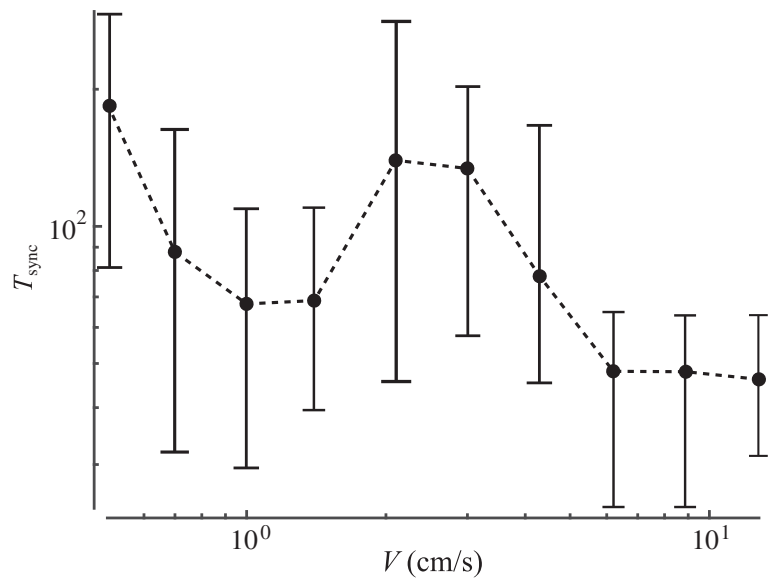

FIG. 12. The time $T_{\text {sync }}$ (in number of cycles) needed to synchronize ten physical robots moving at a speed $V$ in a $60 \times 60 \mathrm{~cm}^{2}$ arena. Markers indicate the average values over five trials. Error bars show the minimum and maximum values over the trials. we observe the emergence of the intermediate synchronization inhibiting regime.

\section{DISCUSSION}

In this paper, we analyzed the dependence of synchronization time on the speed of agents in a population of mobile pulse-coupled oscillators. Previous research showed that synchronization is facilitated by oscillator mobility when the agents interact with others within a certain range. By contrast, if the agents interact with their nearest neighbors, a regime of intermediate speeds is observed where synchronization is hindered. Taking these previous results as a point of departure, we studied various factors that lead to this pernicious effect with a view towards controlling synchronization in real-world applications.

First, the conditions for the previously reported nonmonotonic behavior with nearest-neighbor interaction were studied. We found that the synchronization-hindering regime emerges only with a multiplicative phase-response curve, whereas it is not present in the other studied phaseresponse curves. We devised a metric for the phaseresponse curve, $\Delta r(\phi)$, that correlates with the emergence of the pernicious interval. Indeed, a partial or total blocking of this interval, by means of a refractory period, recovers the monotonic dependence.

We extended the previously studied work by studying $K$ nearest-neighbor and cone-of-vision connectivities with the multiplicative phase-response curve. We linked the previously found monotonic and nonmonotonic synchronization curves by tuning the average size of the agents' neighborhood. For $K$-nearest-neighbor connectivity, as $K$ increases, the effect of the intermediate regime is decreased until a monotonically decreasing dependence is observed. Similarly, for the cone-of-vision connectivity, a synchronization impeding regime appears for small or narrow cones. This nonmonotonic dependence of the synchronization time with agents' speed can be gradually transformed into a monotonic one by widening or extending the range of the cone of vision. The interplay between the cone 
parameters, $\theta$ and $R$, is not trivial; increasing the angle or range of the cone can result in an increased synchronization time. This aspect deserves further analysis in future work.

We analyzed the effect and propagation of small perturbations on initially synchronized systems. It was found that the perturbations are contained within the locally connected cluster and did not spread over the system for the slow regime. In the fast regime, the perturbation propagates to all agents but its magnitude remains small due to the brevity of each interaction. For both slow and fast speeds, a coherent state is achieved in relatively short time. Interestingly, in the intermediate regime, perturbations get amplified as they propagate through the system. Nevertheless, the system will eventually return to synchrony after a certain transient period. We found that the length of this transient increases exponentially with system size. Given the characteristics of this irregular behavior, we draw an analogy with stable chaos. The latter was found in models characterized by discontinuities in the evolution rule [30]. In our case, the firing events and the elicited phase responses constitute such discontinuities.

Similarly to the effect of small perturbations, a system with clock jitter exhibited a strong departure from synchrony in the intermediate regime. Jitter is, to a lesser degree, also deleterious for synchronization if the movement is slow. Consequently, in real applications using the multiplicative phase-response curve, synchronization is stable only for sufficiently high agent speeds.

An important difference between our model and the previous approach on nearest-neighbor interactions [7] is that, in our case, the motion of the units is totally decoupled from the oscillator dynamics. Although not presented here, other motion dynamics were explored with similar results - namely, randomly reorienting units upon firing or upon being influenced by another unit (as in Ref. [7]). Therefore, we observe that the emergence of synchronization regimes depends on the relationship between the temporal dynamics of the oscillators, the spatiotemporal dynamics of the units' motion, and the spatial influence of the neighborhood models.

In practical applications in multiagent systems, such as sensor networks or robotic swarms, both synchronization and desynchronization can serve their purpose to coordinate the agents' activities. The former allows the agents to perform their actions at the same time, whereas the latter allows us to distribute individual activities over time. We observed that the intermediate regime not only slows synchronization down but also promotes desynchronization after small perturbations are applied to the system. Depending on what is most desirable at the time, the agents could switch between a synchronized state or a desynchronized one by changing their speed, modifying their neighborhood scope, increasing or decreasing the refractory period, and/or perturbing the phase of one of them.
In order to test the applicability of our findings in a practical scenario, an experimental study was carried out with a swarm of ten physical robots. These robots communicate the firing of their internal oscillator by flashing onboard lights. These flashings can, in turn, be detected by other robots with their cameras. The physical implementation lead to differences with respect to the simulation, such as finite size of the agents, imperfect signal detection, noninstantaneous firing, etc. Despite the aforementioned challenges, our findings confirm the existence of the nonmonotonic behavior in a swarm of physical robots and the emergence of the intermediate inhibitory regime.

\section{ACKNOWLEDGMENTS}

We thank Matthew J. Doyle for the fruitful discussions, and Stefan M. Trenkwalder for his invaluable help with performing the robotic experiments. This research was supported by the Engineering and Physical Sciences Research Council (Grant No. EP/K031600/1).

[1] S. Strogatz, Sync: The Emerging Science of Spontaneous Order (Hyperion, New York, 2003).

[2] A. Pikovsky, M. Rosenblum, and J. Kurths, Synchronization: A Universal Concept in Nonlinear Sciences (Cambridge University Press, Cambridge, England, 2001).

[3] A. T. Winfree, Biological rhythms and the behavior of populations of coupled oscillators, J. Theor. Biol. 16, 15 (1967).

[4] A. Arenas, A. Díaz-Guilera, J. Kurths, Y. Moreno, and C. Zhou, Biological rhythms and the behavior of populations of coupled oscillators, Phys. Rep. 469, 93 (2008).

[5] R. E. Mirollo and S. H. Strogatz, Synchronization of pulsecoupled biological oscillators, SIAM J. Appl. Math. 50, 1645 (1990).

[6] L. Prignano, O. Sagarra, P. M. Gleiser, and A. Diaz-Guilera, Synchronization of moving integrate and fire oscillators, Int. J. Bifurcation Chaos Appl. Sci. Eng. 22, 1250179 (2012).

[7] L. Prignano, O. Sagarra, and A. Díaz-Guilera, Tuning Synchronization of Integrate-and-Fire Oscillators through Mobility, Phys. Rev. Lett. 110, 114101 (2013).

[8] S. Sarkar and P. Parmananda, Synchronization of an ensemble of oscillators regulated by their spatial movement, Chaos 20, 043108 (2010).

[9] K. Uriu, S. Ares, A. C. Oates, and L. G. Morelli, Dynamics of mobile coupled phase oscillators, Phys. Rev. E 87, 032911 (2013).

[10] L. Janagal and P. Parmananda, Synchronization in an ensemble of spatially moving oscillators with linear and nonlinear coupling schemes, Phys. Rev. E 86, 056213 (2012).

[11] J. Wang, C. Xu, J. Feng, M. Z. Chen, X. Wang, and Y. Zhao, Synchronization in moving pulse-coupled oscillator networks, IEEE Trans. Circuits Syst. I 62, 2544 (2015).

[12] C. Shen, H. Chen, and Z. Hou, Mobility-enhanced signal response in metapopulation networks of coupled oscillators, Europhys. Lett. 102, 38004 (2013). 
[13] C. Shen, H. Chen, and Z. Hou, Mobility and density induced amplitude death in metapopulation networks of coupled oscillators, Chaos 24, 043125 (2014).

[14] J. Gómez-Gardeñes, V. Nicosia, R. Sinatra, and V. Latora, Motion-induced synchronization in metapopulations of mobile agents, Phys. Rev. E 87, 032814 (2013).

[15] F. Perez-Diaz, R. Zillmer, and R. Groß, in Proceedings of the 14th International Conference on Autonomous Agents and Multiagent Systems (AAMAS 2015), Istanbul, 2015, (International Foundation for Autonomous Agents and Multiagent Systems, Richland, SC, 2015), p. 279.

[16] Y. Wang, F. Nunez, and F. J. Doyle, in Proceedings of the American Control Conference (ACC 2014), Portland, OR, 2014 (IEEE, New York, 2014), p. 3553.

[17] N. Fujiwara, J. Kurths, and A. Díaz-Guilera, Synchronization in networks of mobile oscillators, Phys. Rev. E 83, 025101 (2011).

[18] K. Uriu and L. G. Morelli, Collective cell movement promotes synchronization of coupled genetic oscillators, Biophys. J. 107, 514 (2014).

[19] Y. Wang, F. Núñez, and F. J. Doyle III, Energy-efficient pulse-coupled synchronization strategy design for wireless sensor networks through reduced idle listening, IEEE Trans. Signal Process. 60, 5293 (2012).

[20] A. Tyrrell, G. Auer, and C. Bettstetter, in Proceedings of the 1st International Conference on Bio Inspired Models of Network, Information and Computing Systems, Trento (BIONETICS 2006), Italy (ACM, New York, 2006).

[21] A. L. Christensen, R. O'Grady, and M. Dorigo, From fireflies to fault-tolerant swarms of robots, IEEE Trans. Evol. Comput. 13, 754 (2009).

[22] L. E. Parker, in Springer Handbook of Robotics, edited by B. Siciliano and O. Khatib (Springer, New York, 2008), p. 921.

[23] L. Vig and J. A. Adams, Multi-robot coalition formation, IEEE. Trans. Robot. 22, 637 (2006).

[24] P. Ranganathan, R. Morton, A. Richardson, J. Strom, R. Goeddel, M. Bulic, and E. Olson, in Proceedings of the Land Warfare Conference (LWC 2010), Brisbane, 2010.

[25] A. F. T. Winfield, in Distributed Autonomous Robotic Systems 4, edited by L. E. Parker, G. Bekey, and J. Barhen (Springer, New York, 2000), p. 273.
[26] C. Yu, J. Werfel, and R. Nagpal, in Proceedings of the 9th International Conference on Autonomous Agents and Multiagent Systems (AAMAS 2010), Toronto, 2010 (International Foundation for Autonomous Agents and Multiagent Systems, Richland, SC, 2010), p. 1189.

[27] J. Degesys, I. Rose, A. Patel, and R. Nagpal, in Proceedings of the 6th International Conference on Information Processing in Sensor Networks (IPSN 2007), Cambridge, MA, 2007 (ACM, New York, 2007), p. 11.

[28] A. Patel, J. Degesys, and R. Nagpal, in Proceedings of the First International Conference on Self-Adaptive and SelfOrganizing Systems (SASO 2007), Cambridge, MA, 2007 (IEEE, New York, 2007), p. 87.

[29] A. Cornejo and F. Kuhn, in Proceedings of the 24th International Symposium on Distributed Computing (DISC 2010), Cambridge, MA, 2010 (Springer, New York, 2010), p. 148.

[30] A. Politi and A. Torcini, in Nonlinear Dynamics and Chaos: Advances and Perspectives, edited by M. Thiel, J. Kurths, M. C. Romano, G. Károlyi, and A. Moura (Springer, New York, 2010), p. 103.

[31] A. V. Proskurnikov and M. Cao, Synchronization of pulsecoupled oscillators and clocks under minimal connectivity assumptions, IEEE Transactions on Automatic Control (IEEE, 2016).

[32] R. Zillmer, N. Brunel, and D. Hansel, Very long transients, irregular firing, and chaotic dynamics in networks of randomly connected inhibitory integrate-and-fire neurons, Phys. Rev. E 79, 031909 (2009).

[33] F. Perez-Diaz, S. M. Trenkwalder, R. Zillmer, and R. Groß, "Emergence and Inhibition of Synchronization in Robot Swarms," in Proceedings of the 13th International Symposium on Distributed Autonomous Robotic Systems (DARS 2016), London, 2016, edited by R. Groß, S. Berman, E. Frazzoli, M. Gauci, A. Kolling, A. Martinoli, and F. Matsuno (Springer, New York, to be published).

[34] F. Mondada, M. Bonani, X. Raemy, J. Pugh, A. Klaptocz, S. Magnenat, J. C. Zufferey, D. Floreano, and A. Martinoli, in Proceedings of the 9th Conference on Autonomous Robot Systems and Competitions (Robotica 2009) (IPCB-Instituto Politécnico de Castelo Branco, Castelo Branco, Portugal, 2009), p. 59. 